\title{
Raman Spectra and Conformations of Copolypeptides Containing Glycine, L-Alanine, and L-Phenylalanine Residues
}

\author{
Koichi ITOH and Masanao OYA* \\ Department of Chemistry, School of Science and Engineering, \\ Waseda University, Shinjuku-ku, Tokyo 160, Japan \\ * Department of Industrial Chemistry, College of Technology, \\ Gumma University, Kiryu-shi, Gumma 376, Japan
}

(Received May 19, 1986)

\begin{abstract}
Raman spectra were measured for copolymers of $\mathrm{L}$-alanine and glycine and those of L-alanine and L-phenylalanine. The L-alanine-glycine copolypeptides with a glycine residue content of $3-21 \mathrm{~mol} \%$ take the $\alpha$-helical conformation. On incorporation of the glycine residue at more than $30 \%$, the backbone structure changes partly from the $\alpha$-helix to the $\beta$-form. The Lalanine-L-phenylalanine copolypeptides with the L-phenylalanine content of $12-92 \%$ take the $\alpha$ helical structure. The following Raman bands reflect the $\alpha$-helix or the $\beta$-form structure of the component amino acid residues; the bands near 1308, 1108, 530 and $378 \mathrm{~cm}^{-1}$ are characteristic of the L-alanine residue with the $\alpha$-helical structure, the band near $1094 \mathrm{~cm}^{-1}$ of the L-alanine resdue with the $\beta$-form, the band in the $470-480 \mathrm{~cm}^{-1}$ region of the $\mathrm{L}$-phenylalanine residue with the $\alpha$ helix, and the band near $490 \mathrm{~cm}^{-1}$ of the L-phenylalanine with the $\beta$-form.
\end{abstract}

KEY WORDS Raman Specttra / Copolypeptides / Conformation /

The correlation between the positions of the amide I and III bands and the backbone structures of polypeptides such as $\alpha$-helical, $\beta$-form and random coil conformations has been established by a number of previous works. ${ }^{1}$ By using this correlation the Raman spectra of synthetic polypeptides and proteins have been analyzed. Since the skeletal deformation vibrations of chain molecules are sensitive to their conformational changes, the vibrational bands of polypeptides in a lower frequency region (e.g., below $700 \mathrm{~cm}^{-1}$ ) are also expected to give information about their secondary structures. As for the infrared bands, the amide $\mathrm{V}$ mode due to the $\mathrm{NH}$ outof-plane bending vibration $\left(750-600 \mathrm{~cm}^{-1}\right)$ has a correlation with the secondary structures of polypeptide backbones, ${ }^{2}$ and there exist several far-infrared bands which are characteristic of the $\alpha$-helix or the $\beta$-form confor- mation of several amino acid residues., ${ }^{3,4}$ Search for Raman bands characteristic of the secondary structures in the lower frequency region, however, has not so far been done except in a few works. ${ }^{5,6}$ To find Raman bands reflecting the secondary structures and local conformations of amino acid residues, in the present paper, we studied the Raman spectra of copolypeptides containing two kinds of amino acids -i.e., L-alanine and glycine, and L-phenylalanine- with various residue composition ratios.

\section{EXPERIMENTAL}

Copolymers of L-alanine and glycine and those of L-alanine and L-phenylalanine are synthesized with various monomer feed ratios by the $N$-carboxy anhydride method. ${ }^{7,8}$ Acetonitrile and $n$-butyl amine were used as a 


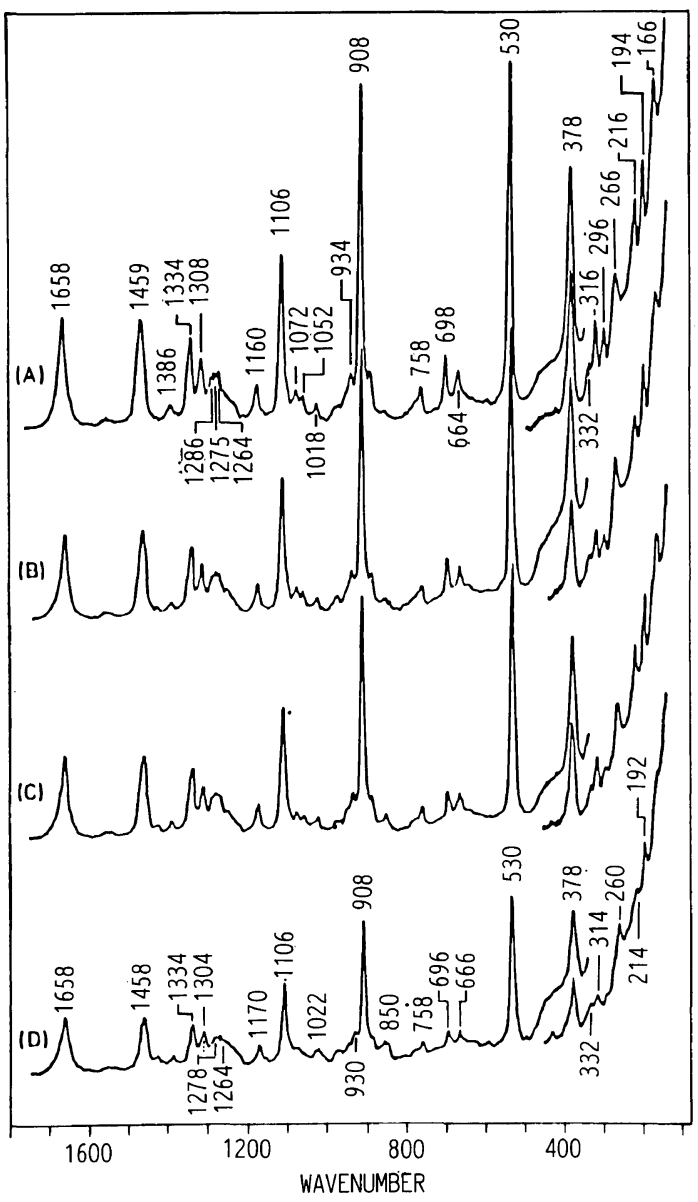

polymerization solvent and as an initiator, respectively. The anhydride to initiator ratio was fixed at about two hundred. The copolymers were obtained by filtration and dried in vacuo at room temperature. The amino acid compositions of the resultant copolymers were determined by the proton NMR spectra of their trifluoroacetic acid- $d_{1}$ solution. The compositions were determined from the peak intensities of signals due to the protons of the methylene (glycine residue), methyl (alanine residue), and phenyl (phenylalanine residue) groups. The accuracy of the compositions thus determined is believed to be within $1 \%$. A sequential polypeptide, poly(L-alanyl-Lphenylalanyl-L-alanine) ((L-Ala-L-Phe-L-

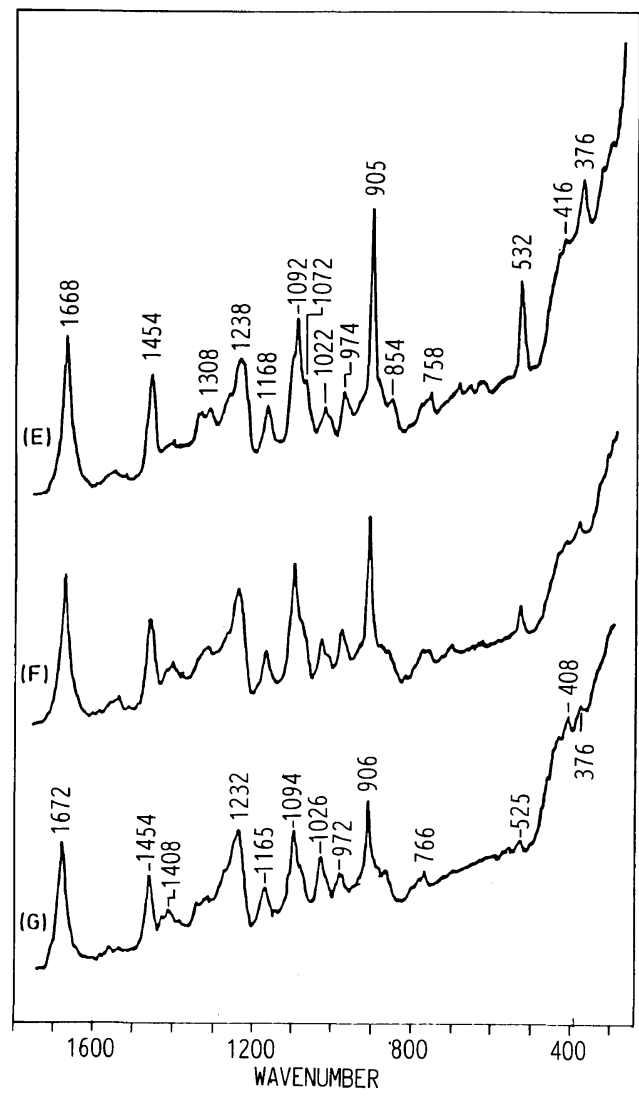

Figure 1. Raman spectra of L-alanine-glycine copolymers in the solid state. Excitation wavelength= $514.5 \mathrm{~nm}$ ( $c a .300 \mathrm{~mW}$ ). Spectral slit width $=5 \mathrm{~cm}^{-1}$. (A) L-alanine residue content $(f(\mathrm{~L}-\mathrm{Ala}))=97 \%$; (B) $f(\mathrm{~L}-\mathrm{Ala})=93 \%$; (C) $f($ L-Ala $)=88 \%$; (D) $f($ L-Ala $)=$ $79 \%$; (E) $f(\mathrm{~L}-\mathrm{Ala})=69 \%$; (F) $f(\mathrm{~L}-\mathrm{Ala})=61 \%$; (G) $f(\mathrm{~L}-\mathrm{Ala})=48 \%$.

Ala), was synthesized following the procedure already reported. ${ }^{4}$

Raman spectra were recorded by the use of a JEOL JRS-400D Raman spectrophotometer equipped with a Spectraphysics model 164 argon ion laser. The 514.5-nm line was used to excite the spectra. Solid samples were packed into a conical depression at the end of a stainless steel rod with a diameter of $3 \mathrm{~mm}$. Raman scattering was collected and introduced to the entrance slit of the spectrometer at an angle of $90^{\circ}$ to the incident excitation light. 


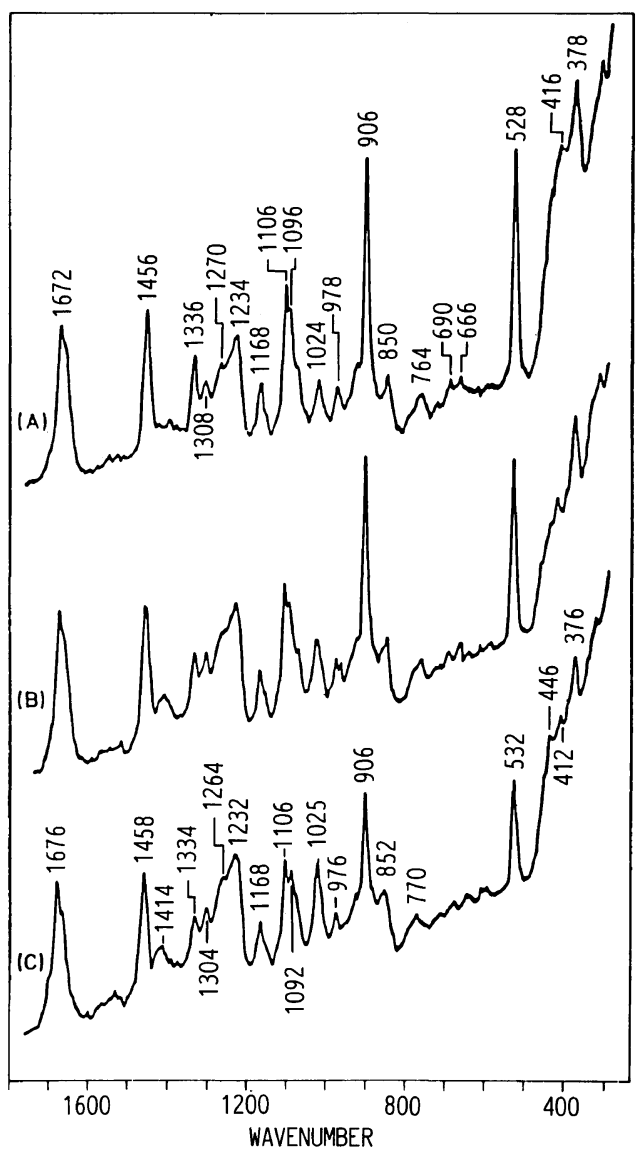

Figure 2. Raman spectra of L-alanine-glycine copolymers treated with dichloroacetic acid (see the text). Excitation wavelength $=514.5 \mathrm{~nm}(c a .300 \mathrm{~mW})$. Spectral slit width $=6 \mathrm{~cm}^{-1}$. (A) L-lalanine residue content $(f(\mathrm{~L}-$ Ala ) $=69 \%$; (B) $f$ (L-Ala) $=61 \%$; (C) $f($ L-Ala $)=48 \%$.

\section{RESULTS AND DISCUSSION}

Figure 1 shows the Raman spectra of copolymers of L-alanine and glycine. As is evident from the spectra in Figures 1(A)-1(D), the copolymers with the L-alanine content of $97-79 \%$ give the amide I band near 1658 $\mathrm{cm}^{-1}$ and the amide III around $1270 \mathrm{~cm}^{-1}$ as a triplet, indicating that the backbone conformation of the copolymers takes the $\alpha$-helix. ${ }^{1}$ The bands near 530 and $378 \mathrm{~cm}^{-1}$ in these spectra correspond to the infrared bands observed in these regions, which have been in- terpreted as characteristic of the L-alanine residue with the $\alpha$-helical conformation. ${ }^{3,4}$ The relative intensity of these bands to the 908 $\mathrm{cm}^{-1}$ one (The latter band is mainly due to the $\mathrm{C}_{\alpha}-\mathrm{C}_{\beta}$ stretching vibration of the L-alanine residue and its intensity is independent of the structures taken by the residue..$^{5}$ ) does not change with the L-alanine residue contents in Figures 1(A)-1(D). Therefore, the $\alpha$-helical backbone conformation, which is mainly composed of L-alanine residues, is not deformed by incorporation of the glycine residues of up to $21 \%$. When the glycine residue content exceeds $30 \%$, however, the backbone conformation changes drastically. This change can be seen from the spectra shown in Figures $1(\mathrm{E})-1(\mathrm{G})$. The amide I band appears at $1668-1672 \mathrm{~cm}^{-1}$ and the amide III at $1238-1232 \mathrm{~cm}^{-1}$, indicating that the samples contain an appreciable amount of the $\beta$ form structure. The relative intensity of the $532-\mathrm{cm}^{-1}$ band to the $905-\mathrm{cm}^{-1}$ one decreases with an increase of the glycine content. From the relative intensity we can estimate the fraction of the L-alanine residues with the $\alpha$ helical conformation relative to the total $\mathrm{L}$ alanine residues to be about 68,17 and $12 \%$ for the samples which give the spectra in Figures 1(E), 1(F), and $1(\mathrm{G})$, respectively. In this estimation we assumed the following points; (i) the relative intensities, $I(530) / I(908)$ and $I(378) / I(908)$, are proportional to the fraction of the $\alpha$-helical L-alanine residues, (ii) all the L-alanine residues of the samples, which give the spectra in Figures 1(A)1(D), take the $\alpha$-helical conformation. The copolypeptides, which give the spectra in Figures 1(D) and 1(E) have an almost identical intrinsic viscosity (about 0.60 in dichloroacetic acid $\left.\left(0.5 \mathrm{~g} \mathrm{dl}^{-1}\right)\right)$ This indicates that they contain a similar number of amino acid residues (about 100). Therefore, the moleclar weight of the samples is not an important factor to explain the above-mentioned conformational changes. The reactivity ratios of the glycine and L-alanine $\mathrm{NCAs}^{7}$ suggest that 


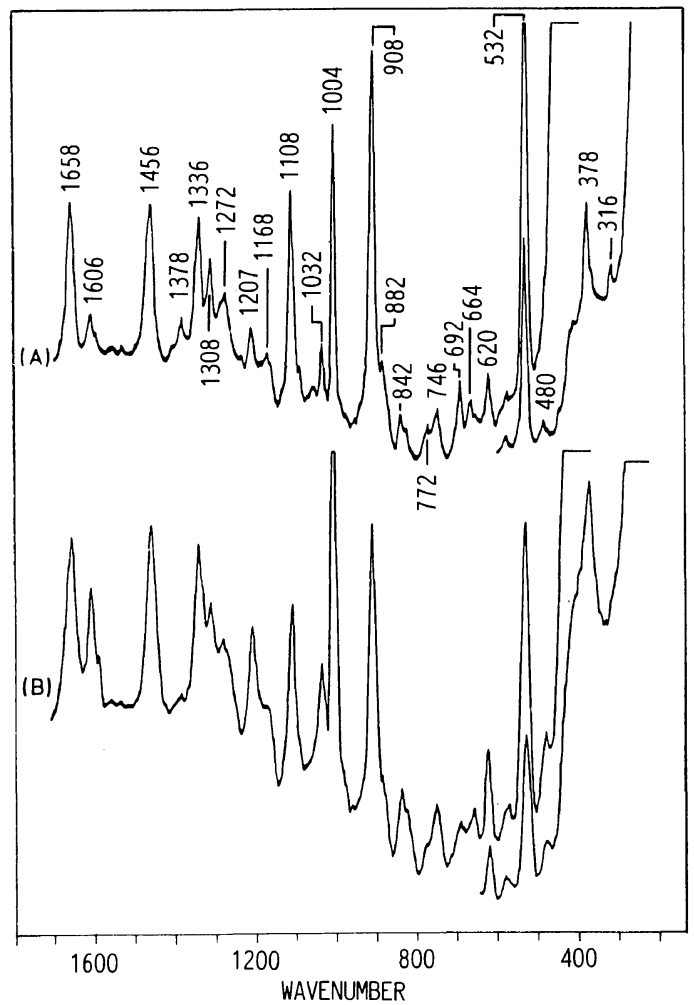

an average block sequence of the L-alanine residues of the copolymer of Figure $1(E)$ is shorter than that of the copolymer of Figure 1(D). Probably, there exists a critical length of the L-alanine residue sequence for the formation of the $\alpha$-helical structure. The abovementioned results indicates that the content of the L-alanine residue sequences longer than the critical length decreases drastically on reduction of the L-alanine content from 79 to $69 \%$. From the spectra in Figure 1, it is also noted that the bands near 1308 and $1106 \mathrm{~cm}^{-1}$ are observed only for the spectra in Figures 1(A)-1(D) while the band at 1094 $\mathrm{cm}^{-1}$ only for those in Figures $1(\mathrm{E})-1(\mathrm{G})$, suggesting that these bands can also be used for the structural diagnosis of the L-alanineglycine copolymers; i.e., the 1308- and 1106$\mathrm{cm}^{-1}$ bands are characteristic of the $\alpha$-helix and the $1094-\mathrm{cm}^{-1}$ of the $\beta$-form.

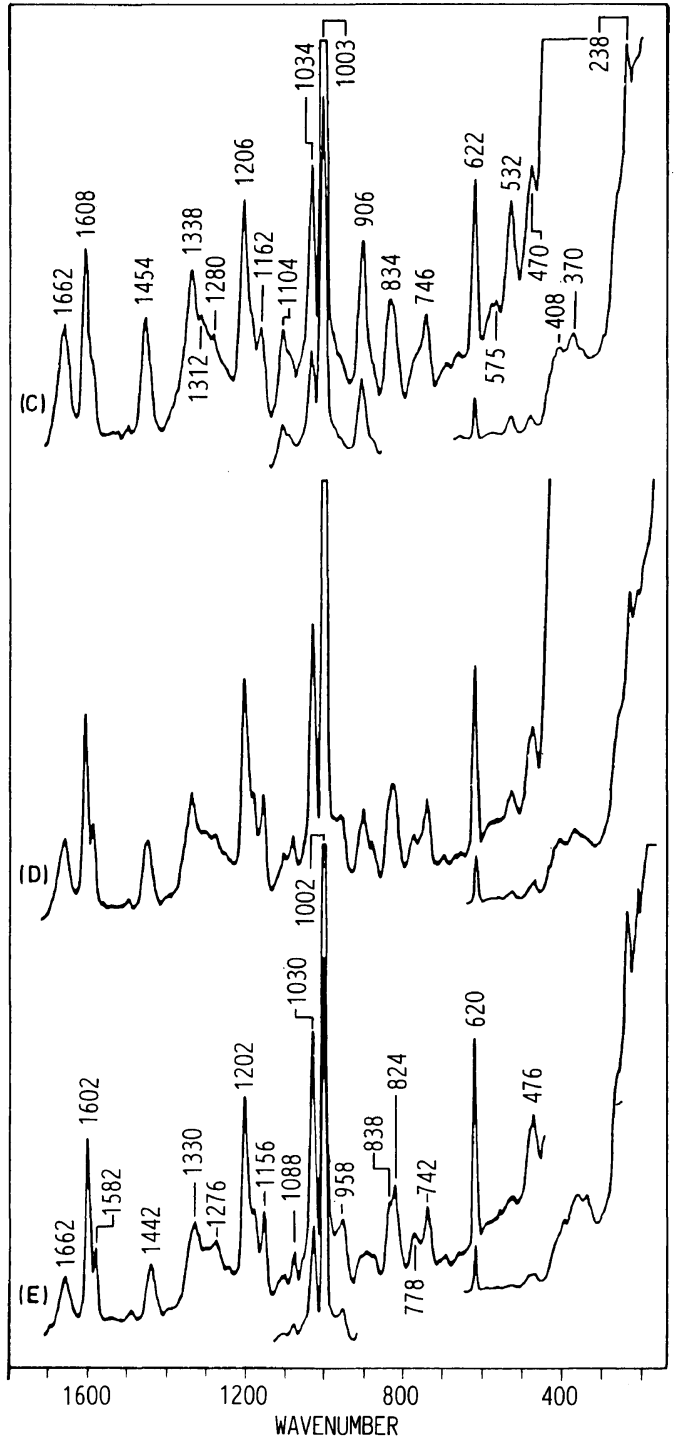

Figure 3. Raman spectra of L-alanine-L-phenylalanine copolymers in the solid state. Excitation wavelength $=514.5 \mathrm{~nm}$ ( $c$ a. $300 \mathrm{~mW})$. Spectral slit width $=7 \mathrm{~cm}^{-1}$. (A) L-alanine residue content $(f(\mathrm{~L}-\mathrm{Ala}))=88 \%$; (B) $f($ L-Ala $)=73 \%$; (C) $f($ L-Ala $)=42 \%$; (D) $f($ L-Ala $)=$ $42 \%$; (D) $f$ (L-Ala) $=23 \%$; (E) $f$ (L-Ala) $=8 \%$.

The copolymers with the L-alanine residue content of 69,61 , and $48 \%$ were dissolved in dichloroacetic acid and reprecipitated by the addition of ethyl ether. After washing the samples repeatedly with ethyl ether, we mea- 


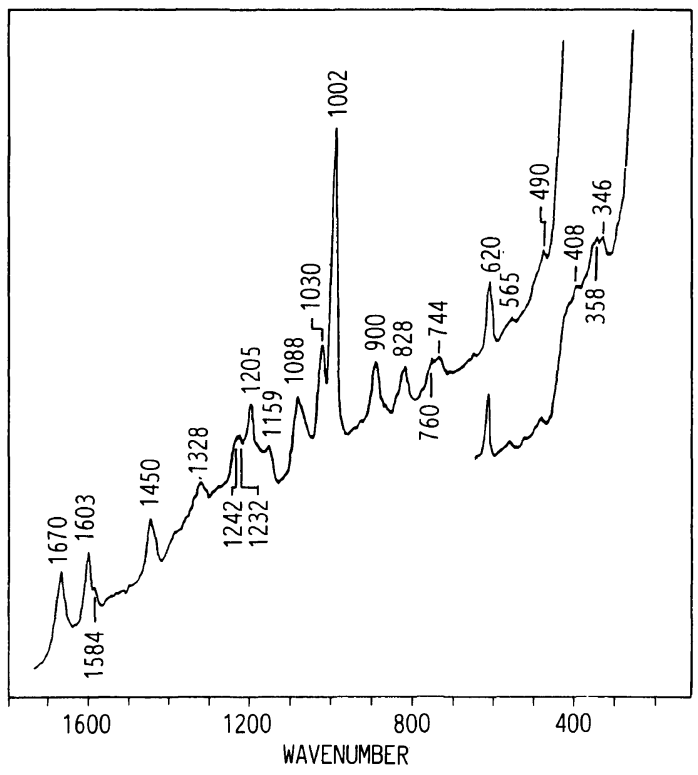

Figure 4. Raman spectra of (L-Ala-L-Phe-L-Ala) in the solid state. Excitation wavelength $=514.5 \mathrm{~nm}(\mathrm{ca}$. $350 \mathrm{~mW}$ ). Spectral slit width $=6 \mathrm{~cm}^{-1}$.

sured their Raman spectra; the results are shown in Figures 2(A)-2(C). Comparison of these spectra with those shown in Figures $1(\mathrm{E})-1(\mathrm{G})$ indicates that the relative intensity of the $530-\mathrm{cm}^{-1}$ band to the $908-\mathrm{cm}^{-1}$ one increases after treatment with dichloroacetic acid. Therefore, the treatment causes an appreciable amount of $\mathrm{L}$-alanine residues to change their structures from the $\beta$-form to the $\alpha$-helix. In addition, the relative intensities of the 1308- and $1106-\mathrm{cm}^{-1}$ bands to that at $1094 \mathrm{~cm}^{-1}$ in Figures 2(A)-2(C) are larger than those in the spectra of the corresponding copolypeptides which are not treated with dichloroacetic acid (Figures 1(E)-1(G)). This supports the above-mentioned conclusion that the former two bands are due to the $\alpha$ helix and the latter the $\beta$-form.

Figures 3(A)-3(E) are the Raman spectra of the copolymers of L-alanine and L-phenylalanine. All the spectra give rise to the amide I at $1658-1662 \mathrm{~cm}^{-1}$ and the amide III at $1272-1280 \mathrm{~cm}^{-1}$, indicating that the copolymers take the $\alpha$-helical structure. The bands near $1308,1108,908,532$, and $378 \mathrm{~cm}^{-1}$, the counterparts of which are observed in the Raman spectra of the L-alanine-glycine copolymers in Figures 1 and 2, are ascribed to the L-alanine residue since they reduce their intensity as the L-alanine content is decreased. As Figure 3 shows, the bands around 1606, $1207,1004,620$, and $480 \mathrm{~cm}^{-1}$ increase their intensity with the L-phenylalanine content, which indicates that all these bands can be assigned to the L-phenylalanine residue. Figure 4 is the Raman spectrum of a sequential polypeptide, (L-Ala-L-Phe-L-Ala $)_{n}$. The infrared spectrum of the same sample shows the amide I bands at 1689 and $1630 \mathrm{~cm}^{-1}$, which can be assigned to the $(\pi, 0)$ and $(0, \pi)$ modes, ${ }^{9,10}$ respectively, of the anti-parallel pleated sheet structure (the $\beta$-form). Corresponding to this result, the sequential polypeptide gives the Raman bands due to the amide I and III modes at 1670 and $1235 \mathrm{~cm}^{-1}$, respectively, which is a spectral feature characteristic of the $\beta$-form. ${ }^{1}$ A comparison of the spectrum in Figure 4 with those in Figures 3(B) and 3(C) indicates that the bands near $1308,1108,532$, and $378 \mathrm{~cm}^{-1}$ in the latter spectra are absent in the former, which confirms that these bands are characteristic of the $\alpha$-helical structure of the L-alanine residue. The bands near 1606, 1207, 1004, and $620 \mathrm{~cm}^{-1}$ due to the $\mathrm{L}$-phenylalanine residue in Figure 3 are found also in the spectrum of the sequential polypeptide with the $\beta$-form structure, which indicates that these bands do not reflect the backbone conformation. The well-defined band at $490 \mathrm{~cm}^{-1}$ in Figure 4 corresponds to the band of the $480-470 \mathrm{~cm}^{-1}$ region in Figures 3(A)-3(E). A frequency shift of this band to a higher frequency side can be taken as an indication of the conformational change of the L-phenylalanine residue from the $\alpha$-helical structure to the $\beta$ form.

The Raman scattering bands reflecting the $\alpha$-helical and $\beta$-form structures of $\mathrm{L}$-alanine and L-phenylalanine residues are summarized 
Table I. Frequencies $\left(\mathrm{cm}^{-1}\right)$ of Raman scattering bands reflecting the $\alpha$-helical and $\beta$-from structures of amino acid residues

\begin{tabular}{lll}
\hline $\begin{array}{c}\text { Amino acid } \\
\text { residues }\end{array}$ & $\alpha$-Helix (right-handed) & $\beta$-Form \\
\hline L-Alanine & $\begin{array}{l}1308-1304,1108-1104, \\
\end{array}$ & $525-532,378-376$ \\
& $470-480$ & 490 \\
L-Phenylalanine & 470 & \\
L-Valine $^{\mathbf{a}}$ & 545,430 & \\
D-Alanine $^{\mathrm{b}}$ & 478 & \\
\hline
\end{tabular}

a Taken from ref 6 .

b Taken from ref 5 .

in Table $I$ in which the Raman band characteristic of the right-handed $\alpha$-helical structure of $\mathrm{L}$-valine and $\mathrm{D}$-alanine residues ${ }^{5,6}$ are also included. These results are very useful to elucidate the local conformations of the component amino acid residues of polypeptides and proteins.

\section{REFERENCES}

1. B. G. Frushour and J. L. Koenig, "Advances in Infrared and Raman Spectroscopy," Vol. 1, R. J. H. Clark and R. E. Hester, Ed., Heyden, 1975, p 35.

2. T. Miyazawa, K. Fukushima, S. Sugano, and Y. Masuda, "Conformations of Biopolymers," Vol. II, G. N. Ramachandran, Ed., Academic Press., New York., N. Y., 1967, p 557.

3. K. Itoh, H. Katabuchi, and T. Shimanouchi, Nature New Biology, 239, 42 (1972).

4. K. Itoh and H. Katabuchi, Biopolymers, 12, 921 (1973).

5. K. Itoh, H. Hinomoto, and T. Shimanouchi, Biopolymers, 13, 307 (1978).

6. G. D. Fasman, K. Itoh, C. S. Liu, and R. C. Rord, Biopolymers, 17, 125 (1978).

7. M. Oya, K. Uno, and Y. Iwakura, J. Polym. Sci., A1, 10, 613 (1972)

8. M. Oya and T. Takahashi, J. Polym. Sci., Polym. Chem. Ed., 20, 529 (1982).

9. T. Miyazawa, J. Chem. Phys., 32, 1647 (1960).

10. T. Miyazawa and E. R. Blout, J. Am. Chem. Soc., 83, 712 (1961). 